\title{
EXTREMAL CHARACTERIZATIONS OF ASPLUND SPACES
}

\author{
BORIS S. MORDUKHOVICH AND YONGHENG SHAO
}

(Communicated by Dale Alspach)

\begin{abstract}
We prove new characterizations of Asplund spaces through certain extremal principles in nonsmooth analysis and optimization. The latter principles provide necessary conditions for extremal points of set systems in terms of Fréchet normals and $\varepsilon$-normals.
\end{abstract}

\section{INTRODUCTION}

In [1], Asplund introduced a class of Banach spaces (later called Asplund spaces) on which every continuous convex function is Fréchet differentiable at a dense set of points. This class turns out to be very important for the geometric theory of Banach spaces as well as for numerous applications. We refer to [4] and [17] and bibliographies therein for more information.

It is well known that the class of Asplund spaces is rich enough including, among others, every Banach space with separable dual and every space with an equivalent Fréchet differentiable renorm (in particular, all reflexive spaces). Moreover, there are Asplund spaces of continuous functions where every equivalent norm fails to be even Gâteaux differentiable at some nonzero points.

Now there are many characterizations of Asplund spaces in terms of structural properties of Banach spaces which may not be directly related to differentiability. One of the most useful characterizations is as follows: A Banach space is Asplund if and only if each of its separable subspaces has a separable dual; see $[4,17]$.

On the other hand, Fabian $[6,7]$ developing a work of Ioffe [8], obtained characterizations of Asplund spaces in terms of Fréchet subdifferentiability of nonconvex lower semicontinuous (l.s.c.) functions. This links Asplund spaces to general constructions of nonsmooth analysis. Note that progress in that direction was achieved on the basis of variational principles of Ekeland [5] and Borwein-Preiss [2].

In this paper, we obtain two new characterizations of Asplund spaces which directly relate those to basic principles in nonsmooth optimization. Namely, we prove that Asplundity of a Banach space is equivalent to the fulfillment of (generalized) Euler equations for extremal points of closed set systems expressed in terms of Fréchet normals and $\varepsilon$-normals.

The results obtained have many useful applications to nonsmooth analysis and optimization as well as to geometry of Banach spaces. In particular, they easily

Received by the editors February 22, 1994, and, in revised form, August 1, 1994.

1991 Mathematics Subject Classification. Primary 46B20; Secondary 49J52.

This research was partially supported by the National Science Foundation under grants DMS9206989 and DMS-9404128. 
imply a nonconvex analogue of the celebrated Bishop-Phelps theorem in Asplund spaces; see Section 3. We refer the reader to our paper [16] for more information.

\section{Constructions in NONSmooth AnAlysis}

Everywhere in this paper $X$ is a Banach space and $X^{*}$ is its dual with the same notation for norms $\|\cdot\|$. We denote by $B$ and $B^{*}$ the unit closed balls in the spaces $X$ and $X^{*}$, respectively, and by $A^{*}$ the adjoint operator to a linear continuous operator $A$. The symbol $B_{r}(x)$ stands for the closed ball with center $x$ and radius $r$.

Let $\Omega$ be a nonempty and closed subset of $X$, and let $\varepsilon \geq 0$. Given $\bar{x} \in \Omega$, the nonempty set

$$
\widehat{N}_{\varepsilon}(\bar{x} ; \Omega):=\left\{x^{*} \in X^{*} \mid \limsup _{x(\in \Omega) \rightarrow \bar{x}} \frac{\left\langle x^{*}, x-\bar{x}\right\rangle}{\|x-\bar{x}\|} \leq \varepsilon\right\}
$$

is called the set of Fréchet $\varepsilon$-normals to $\Omega$ at $\bar{x}$. When $\varepsilon=0$, the set (2.1) is a cone which is called the Fréchet normal cone to $\Omega$ at $\bar{x}$ and is denoted by $\widehat{N}(\bar{x} ; \Omega)$.

Note that for any $\varepsilon \geq 0$, the set (2.1) is convex but may not be weak-star closed. If $\Omega$ is convex, then

$$
\widehat{N}_{\varepsilon}(\bar{x} ; \Omega)=\left\{x^{*} \in X^{*} \mid\left\langle x^{*}, x-\bar{x}\right\rangle \leq \varepsilon\|x-\bar{x}\| \quad \text { for all } x \in \Omega\right\} \quad \forall \varepsilon \geq 0,
$$

which implies that the Fréchet normal cone $\widehat{N}(\bar{x} ; \Omega)$ is a generalization of the normal cone of convex analysis. For general sets $\Omega$ one obviously has

$$
\widehat{N}_{\varepsilon}(\bar{x} ; \Omega) \supset \widehat{N}(\bar{x} ; \Omega)+\varepsilon B^{*} \quad \forall \varepsilon \geq 0 .
$$

Observe also the useful property

$$
\widehat{N}\left(\left(\bar{x}_{1}, \bar{x}_{2}\right) ; \Omega_{1} \times \Omega_{2}\right)=\widehat{N}\left(\bar{x}_{1} ; \Omega_{1}\right) \times \widehat{N}\left(\bar{x}_{2} ; \Omega_{2}\right) \quad \forall\left(\bar{x}_{1}, \bar{x}_{2}\right) \in \Omega_{1} \times \Omega_{2},
$$

valid for any closed sets $\Omega_{i} \subset X_{i}$ in Banach spaces $X_{i}, i=1,2$.

Let $\varphi: X \rightarrow(-\infty, \infty]$ be an extended-real-valued l.s.c. function and let $\bar{x} \in$ $\operatorname{dom} \varphi$. For any $\varepsilon \geq 0$ we define the convex set

$$
\widehat{\partial}_{\varepsilon} \varphi(\bar{x}):=\left\{x^{*} \in X^{*} \mid\left(x^{*},-1\right) \in \widehat{N}_{\varepsilon}((\bar{x}, \varphi(\bar{x})) ; \operatorname{epi} \varphi)\right\}
$$

which is called the Fréchet $\varepsilon$-subdifferential of $\varphi$ at $\bar{x}$. If $\varepsilon=0$ in (2.4), then this set is called the Fréchet subdifferential and is denoted by $\widehat{\partial} \varphi(\bar{x})$.

The Fréchet subdifferential defined is a generalization of the classical Fréchet derivative $\varphi^{\prime}(\bar{x})$. This means that if $\varphi$ is Fréchet differentiable at $\bar{x}$, then $\widehat{\partial} \varphi(\bar{x})$ is a singleton with $\widehat{\partial} \varphi(\bar{x})=\left\{\varphi^{\prime}(\bar{x})\right\}$. For the case of convex functions, $\widehat{\partial} \varphi(\bar{x})$ coincides with the subdifferential of convex analysis.

It is easy to check the following analogue of the Fermat stationary principle in the general nonsmooth setting:

$$
0 \in \widehat{\partial} \varphi(\bar{x}) \text { for any local minimizer } \bar{x} \in \operatorname{dom} \varphi .
$$

In some situations it is more convenient to replace $\widehat{\partial}_{\varepsilon} \varphi(\bar{x})$ in $(2.4)$ by its analytic counterpart:

$$
\widetilde{\partial}_{\varepsilon} \varphi(\bar{x}):=\left\{x^{*} \in X^{*} \mid \liminf _{x \rightarrow \bar{x}} \frac{\varphi(x)-\varphi(\bar{x})-\left\langle x^{*}, x-\bar{x}\right\rangle}{\|x-\bar{x}\|} \geq-\varepsilon\right\}, \quad \varepsilon \geq 0 .
$$


For any closed set $\Omega$ and $\bar{x} \in \Omega$, one obviously has

$$
\widetilde{\partial}_{\varepsilon} \delta(\bar{x}, \Omega)=\widehat{N}_{\varepsilon}(\bar{x}, \Omega) \quad \forall \varepsilon \geq 0
$$

where $\delta(\cdot, \Omega)$ is the indicator of $\Omega$, i.e., $\delta(x, \Omega)=0$ for $x \in \Omega$ and $\delta(x, \Omega)=\infty$ for $x \notin \Omega$.

The proof of the next proposition follows from the definitions and can be found, e.g., in [9, Proposition 1].

2.1. Proposition. For any $\varepsilon \geq 0$ one has $\widetilde{\partial}_{\varepsilon} \varphi(\bar{x}) \subset \widehat{\partial}_{\varepsilon} \varphi(\bar{x})$ and

$$
\left[x^{*} \in \widehat{\partial}_{\varepsilon} \varphi(\bar{x})\right] \Rightarrow\left[x^{*} \in \widetilde{\partial}_{\varepsilon_{1}} \varphi(\bar{x})\right] \text { with } \varepsilon_{1}=(\varepsilon /(1-\varepsilon))\left(1+\left\|x^{*}\right\|\right) .
$$

In particular, $\widehat{\partial} \varphi(\bar{x})=\widetilde{\partial}_{0} \varphi(\bar{x})$.

Now let us present two important subdifferential characterizations of Asplund spaces proved by Fabian $[6,7]$.

2.2. Proposition. Let $X$ be a Banach space. Then the following are equivalent:

(a) $X$ is an Asplund space.

(b) For any $\varepsilon>0$ and $\delta>0$, any functions $\varphi_{1}, \ldots, \varphi_{n}: X \rightarrow(-\infty, \infty], n \geq 2$, and any $\bar{x} \in \operatorname{dom} \varphi_{1} \cap \ldots \cap \operatorname{dom} \varphi_{n}$ such that $\varphi_{1}$ is l.s.c. and $\varphi_{2}, \ldots, \varphi_{n}$ are Lipschitz continuous around $\bar{x}$, one has

$$
\begin{aligned}
\widetilde{\partial}_{\varepsilon}\left(\varphi_{1}+\ldots+\varphi_{n}\right)(\bar{x}) \subset \bigcup\left\{\widetilde{\partial}_{\varepsilon} \varphi_{1}\left(x_{1}\right)+\ldots+\widetilde{\partial}_{\varepsilon} \varphi_{n}\left(x_{n}\right) \mid x_{i} \in B_{\delta}(\bar{x}),\right. \\
\\
\left.\left|\varphi_{i}\left(x_{i}\right)-\varphi_{i}(\bar{x})\right| \leq \delta, i=1, \ldots, n\right\} .
\end{aligned}
$$

(c) For any $\varepsilon \geq 0, \delta>0, \gamma>0$, any functions $\varphi_{1}, \ldots, \varphi_{n}: X \rightarrow(-\infty, \infty], n \geq$ 2 , and any $\bar{x} \in \operatorname{dom} \varphi_{1} \cap \ldots \cap \operatorname{dom} \varphi_{n}$ such that $\varphi_{1}$ is l.s.c. and $\varphi_{2}, \ldots, \varphi_{n}$ are Lipschitz continuous around $\bar{x}$, one has

$$
\begin{aligned}
\widetilde{\partial}_{\varepsilon}\left(\varphi_{1}+\ldots+\varphi_{n}\right)(\bar{x}) \subset \bigcup\{ & \left\{\varphi_{1}\left(x_{1}\right)+\ldots+\widehat{\partial} \varphi_{n}\left(x_{n}\right) \mid x_{i} \in B_{\delta}(\bar{x}),\right. \\
& \left.\left|\varphi_{i}\left(x_{i}\right)-\varphi_{i}(\bar{x})\right| \leq \delta, i=1, \ldots, n\right\}+(\varepsilon+\gamma) B^{*} .
\end{aligned}
$$

Assertions (b) and (c) mean, respectively, that Asplundity of any Banach space $X$ is equivalent to "fuzzy calculus" (2.8) for Fréchet $\varepsilon$-subdifferentials as $\varepsilon>0$ and that calculus (2.9) including $\varepsilon=0$. The following corollary of (c) and (2.7) is useful.

2.3. Corollary. Let $\Omega$ be a closed subset of the Asplund space $X$ and let $\bar{x} \in \Omega$. Then for any $\varepsilon \geq 0, \delta>0$, and $\gamma>0$ one has

$$
\widehat{N}_{\varepsilon}(\bar{x} ; \Omega) \subset \bigcup\left\{\widehat{N}(x ; \Omega) \mid x \in B_{\delta}(\bar{x}) \cap \Omega\right\}+(\varepsilon+\gamma) B^{*} .
$$

\section{Extremal Characterizations}

Let $\Omega_{1}, \Omega_{2}, \ldots, \Omega_{n}$ be nonempty closed subsets of a Banach space $X$ and let $\bar{x} \in \Omega_{1} \cap \ldots \cap \Omega_{n}$. Following $[11,14]$, we say that $\bar{x}$ is a locally extremal point of the set system $\left\{\Omega_{1}, \ldots, \Omega_{n}\right\}$ if there are sequences $\left\{a_{i k}\right\} \subset X, i=1, \ldots, n$, and $\mathrm{a}$ neighborhood $U$ of $\bar{x}$ such that $a_{i k} \rightarrow 0$ as $k \rightarrow \infty$ for any $i=1, \ldots, n$ and

$$
\bigcap_{i=1}^{n}\left\{\Omega_{i}-a_{i k}\right\} \cap U=\emptyset \quad \forall k=1,2, \ldots
$$

We say that sets $\Omega_{1}, \ldots, \Omega_{n}(n \geq 2)$ generate a (locally) extremal system $\left\{\Omega_{1}, \ldots, \Omega_{n}\right\}$ if they have at least one locally extremal point. 
One can see that this geometric concept of set extremality covers conventional notions of optima in problems of scalar and/or vector optimization with constraints. We refer to [10-15] for various examples and discussions. A simple example of such an extremal system is provided by the pair $\{\bar{x}, \Omega\}$ where $\bar{x}$ is a boundary point of the closed set $\Omega$. There are also close connections between extremality and separability for systems of convex and nonconvex sets; see [14, Section 6].

Now let us consider dual characteristics of locally extremal points of closed set systems in terms of Fréchet normals and $\varepsilon$-normals.

3.1. Definition. Let $X$ be a Banach space. We say that the $F_{\varepsilon}$-extremal principle holds in $X$ if for any extremal system of closed sets $\Omega_{i} \subset X, i=1, \ldots, n(n \geq 2)$, any locally extremal point $\bar{x}$ of $\left\{\Omega_{1}, \ldots, \Omega_{n}\right\}$, and any $\varepsilon>0$ there exist $x_{i} \in \Omega_{i} \cap B_{\varepsilon}(\bar{x})$ and $x_{i}^{*} \in X^{*}$ such that

$$
\begin{gathered}
x_{i}^{*} \in \widehat{N}_{\varepsilon}\left(x_{i} ; \Omega_{i}\right) \quad \text { for } i=1, \ldots, n, \\
x_{1}^{*}+x_{2}^{*}+\ldots+x_{n}^{*}=0, \\
\left\|x_{1}^{*}\right\|+\left\|x_{2}^{*}\right\|+\ldots+\left\|x_{n}^{*}\right\|=1 .
\end{gathered}
$$

If (3.1) in the above relationships is replaced by

$$
x_{i}^{*} \in \widehat{N}\left(x_{i} ; \Omega_{i}\right)+\varepsilon B^{*} \quad \text { for } i=1, \ldots, n,
$$

then we say that the $F$-extremal principle holds in $X$.

Relationships (3.1)-(3.4) provide necessary conditions for extremality of set systems and appear to be generalized Euler equations in the abstract geometric framework. They naturally imply necessary optimality conditions in various problems of constrained optimization. In finite dimensions, both relationships (3.1) and (3.4) are equivalent to

$$
x_{i}^{*} \in N\left(\bar{x} ; \Omega_{i}\right) \text { for } i=1, \ldots, n
$$

where the nonconvex normal cone $N(\bar{x} ; \Omega)$ is the Kuratowski-Painlevé upper limit of both $\widehat{N}(x ; \Omega)$ and $\widehat{N}_{\varepsilon}(x, \Omega)$ as $x \rightarrow \bar{x}$ and $\varepsilon \downarrow 0$. Such an extremal principle was at the heart of the geometric approach to nonsmooth analysis and optimization developed in Mordukhovich [12-15].

In infinite dimensions, the $F_{\varepsilon}$-extremal principle was first proved by Kruger and Mordukhovich [11] for the case of Banach spaces having Fréchet differentiable renorms with help of Ekeland's variational principle; see also [10,14]. In the same setting, Ioffe has observed for $n=2$ (see [9, proof of Lemma 2]) that one can replace (3.1) by (3.4) using the Borwein-Preiss smooth variational principle instead of Ekeland's result. To obtain the limiting form (3.2), (3.5) of the extremal principle in infinite dimensions (with the sequential weak-star convergence on $X^{*}$ in the definition of $N(\bar{x} ; \Omega)$ ), we should impose some additional Lipschitz-like assumptions on all but one sets $\Omega_{i}$; cf. $[10,11,14,16]$.

By virtue of (2.2), one can conclude that the $F$-extremal principle implies its $F_{\varepsilon^{-}}$ counterpart in any Banach space. The opposite is also true for the case of Asplund spaces due to Corollary 2.3. The following main result of this paper establishes that these extremal principles hold in any Asplund space and, moreover, Asplundity of a Banach space $X$ is equivalent to the fulfillment of each of these principles in $X$. 
3.2. Theorem. Let $X$ be a Banach space. Then the following are equivalent:

(i) $X$ is an Asplund space.

(ii) The F-extremal principle holds in $X$.

(iii) The $F_{\varepsilon}$-extremal principle holds in $X$.

The proof of this theorem will be presented in the next section. From Theorem 3.2 and Proposition 2.2, one immediately gets the following corollary.

3.3. Corollary. Let $X$ be a Banach space. Then the F-extremal principle and $F_{\varepsilon}$-extremal principle are equivalent and hold in $X$ if and only if any of the "fuzzy calculi" (2.8) and (2.9) hold in this space.

Next let us present an easy application of the $F$-extremal principle to geometry of Banach spaces. The following corollary can be treated as a nonconvex generalization of the well-known Bishop-Phelps theorem (see [17, Theorem 3.18]) in Asplund spaces. We say that $x \in \Omega$ is a F-proper point of $\Omega$ if $\widehat{N}(x ; \Omega) \neq\{0\}$; cf. Borwein and Strojwas [3].

3.4. Corollary. Let $X$ be an Asplund space and let $\Omega$ be a closed subset. Then the set of $F$-proper points of $\Omega$ is dense in the boundary of $\Omega$.

Proof. Let $\bar{x}$ be a boundary point of the set $\Omega$. Then it is a locally extremal point of the systems $\left\{\Omega_{1}, \Omega_{2}\right\}$ where $\Omega_{1}:=\Omega$ and $\Omega_{2}:=\{\bar{x}\}$. According to Theorem 3.2, one can use the $F$-extremal principle. Applying this result with $\varepsilon \in(0,1 / 2)$, we find $z \in \Omega$ and $x_{1}^{*}, x_{2}^{*} \in X^{*}$ such that $\|z-\bar{x}\| \leq \varepsilon$ and

$$
x_{1}^{*} \in \widehat{N}(z ; \Omega)+\varepsilon B^{*}, \quad\left\|x_{1}^{*}\right\|+\left\|x_{2}^{*}\right\|=1, \quad x_{1}^{*}+x_{2}^{*}=0 .
$$

This implies $\left\|x_{1}^{*}\right\|=1 / 2>\varepsilon$ and ensures that $\widehat{N}(z ; \Omega)$ contains a nonzero element. Therefore, $z \in B_{\varepsilon}(\bar{x})$ is a $F$-proper boundary point of $\Omega$. This proves the corollary.

Note that for the case of two sets $\left\{\Omega_{1}, \Omega_{2}\right\}$, the extremal principles in Theorem 3.2 may be viewed as nonconvex generalizations of the classical separation theorem with no interiority-like assumptions.

\section{Proof of the theorem}

The proof of the theorem is based on the following two lemmas which are certainly of independent interest. The first lemma provides a general setting where "fuzzy calculus" (2.9) for Fréchet subdifferentials implies the $F$-extremal principle.

4.1. Lemma. Let $X$ be a Banach space, and let "fuzzy calculus" (2.9) be fulfilled with $\varepsilon=0$ for any l.s.c. functions on $X^{2}$ all but one of them being locally Lipschitzian. Then the F-extremal principle holds for locally extremal points of any extremal system of two closed sets in $X$.

Proof. Let $\Omega_{1}$ and $\Omega_{2}$ be two closed sets in $X$ which form an extremal system, and let $\bar{x} \in \Omega_{1} \cap \Omega_{2}$ be a locally extremal point of $\left\{\Omega_{1}, \Omega_{2}\right\}$. We are going to prove that for any $\varepsilon>0$ there exist $x_{i} \in \Omega_{i} \cap B_{\varepsilon}(\bar{x})$ and $x_{i}^{*} \in \widehat{N}\left(x_{i} ; \Omega_{i}\right)+\varepsilon B^{*}, i=1,2$, such that

$$
\left\|x_{1}^{*}\right\|+\left\|x_{2}^{*}\right\|=1 \quad \text { and } \quad x_{1}^{*}+x_{2}^{*}=0 .
$$


According to the definition of locally extremal points, for given $\varepsilon>0$ we can choose $a \in X$ such that $\|a\|<\varepsilon^{2} / 2$ and $\left(\Omega_{1}+a\right) \cap \Omega_{2} \cap U=\emptyset$ for some neighborhood $U$ of $\bar{x}$. For simplicity we take $U=X$. Thus considering the function

$$
f(u, v):=\|u-v+a\| / 2,
$$

we conclude that $f(u, v)>0$ for any $u \in \Omega_{1}$ and $v \in \Omega_{2}$ while $f(\bar{x}, \bar{x})<(\varepsilon / 2)^{2}$.

Now let us apply Ekeland's variational principle [5] to the function $f$ on the complete metric space $E:=\Omega_{1} \times \Omega_{2}$ whose metric is induced by the norm $\|(u, v)\|:=$ $\|u\|+\|v\|$ on $X^{2}$. Using this result with $\varepsilon:=(\varepsilon / 2)^{2}$ and $\lambda:=\varepsilon / 2$, we find points $\left(\tilde{x}_{1}, \tilde{x}_{2}\right) \in E$ such that $\left\|\tilde{x}_{i}-\bar{x}\right\| \leq \lambda=\varepsilon / 2, i=1,2$, and

$$
f\left(\tilde{x}_{1}, \tilde{x}_{2}\right) \leq f(u, v)+(\varepsilon / 2)\left(\left\|u-\tilde{x}_{1}\right\|+\left\|v-\tilde{x}_{2}\right\|\right)
$$

for all $(u, v) \in E$. This means that the function

$$
\varphi(u, v):=f(u, v)+(\varepsilon / 2)\left(\left\|u-\tilde{x}_{1}\right\|+\left\|v-\tilde{x}_{2}\right\|\right)+\delta\left((u, v), \Omega_{1} \times \Omega_{2}\right)
$$

on $X^{2}$ attains its (unconditional) local minimum at $\left(\tilde{x}_{1}, \tilde{x}_{2}\right)$. Therefore, $0 \in$ $\widehat{\partial} \varphi\left(\tilde{x}_{1}, \tilde{x}_{2}\right)$ by virtue of $(2.5)$.

Let us employ "fuzzy calculus" (2.9) with $\varepsilon=0$ and $\delta=\gamma=\varepsilon / 2$ for the sum of functions in (4.3). This gives us pairs $\left(x_{1}, x_{2}\right) \in \Omega_{1} \times \Omega_{2}$ and $\left(y_{1}, y_{2}\right) \in X^{2}$ with $\left\|x_{i}-\tilde{x}_{i}\right\| \leq \varepsilon / 2$ and $\left\|y_{i}-\tilde{x}_{i}\right\| \leq \varepsilon / 2, i=1,2$, such that $f\left(y_{1}, y_{2}\right)>0$ and

$$
0 \in \widehat{\partial} f\left(y_{1}, y_{2}\right)+\varepsilon\left(B^{*} \times B^{*}\right)+\widehat{N}\left(x_{1} ; \Omega_{1}\right) \times \widehat{N}\left(x_{2} ; \Omega_{2}\right)
$$

by virtue of (2.3) and (2.7). Now using the well-known subdifferentiation formula for the norm function (4.2) at nonzero points, one can reduce (4.4) to the inclusion

$$
0 \in\left(x^{*} / 2,-x^{*} / 2\right)+\varepsilon\left(B^{*} \times B^{*}\right)+\widehat{N}\left(x_{1} ; \Omega_{1}\right) \times \widehat{N}\left(x_{2} ; \Omega_{2}\right)
$$

with $\left\|x^{*}\right\|=1$. Letting $x_{1}^{*}:=-x^{*} / 2$ and $x_{2}^{*}:=x^{*} / 2$, we get elements $x_{i}^{*} \in$ $\widehat{N}\left(x_{i} ; \Omega_{i}\right)+\varepsilon B^{*}$ satisfying (4.1). This ends the proof of Lemma 4.1.

Now let us show that the $F_{\varepsilon}$-extremal principle in $X \times \mathbf{R}$ implies the following crucial case of "fuzzy calculus" (2.8) for Fréchet $\varepsilon$-subdifferentials $(\varepsilon>0)$ in $X$.

4.2. Lemma. Let $X$ be a Banach space, and let the $F_{\varepsilon}$-extremal principle hold for any extremal system of two closed sets in $X \times \mathbf{R}$. Let $\varphi_{1}$ and $\varphi_{2}$ be functions from $X$ into $(-\infty, \infty]$ such that $\varphi_{1}$ is Lipschitz continuous, $\varphi_{2}$ is l.s.c. around $\bar{x}$, and the sum $\varphi_{1}+\varphi_{2}$ attains its local minimum at $\bar{x}$. Then for any $\varepsilon>0$ and $\delta>0$ there exist $x_{i} \in B_{\delta}(\bar{x})$ with $\left|\varphi_{i}\left(x_{i}\right)-\varphi_{i}(\bar{x})\right| \leq \delta, i=1,2$, such that

$$
0 \in \widetilde{\partial}_{\varepsilon} \varphi_{1}\left(x_{1}\right)+\widetilde{\partial}_{\varepsilon} \varphi_{2}\left(x_{2}\right) .
$$

Proof. Fix $\varepsilon>0$ and $\delta>0$ such that $\varphi_{1}$ is Lipschitz continuous on $B_{\delta}(\bar{x})$ with constant $L \geq 0$. For further discussions we choose a number $\gamma>0$ satisfying

$$
\gamma<\min \left\{\frac{1}{2(2 L+3)}, \frac{\varepsilon}{4(L+1)(2 L+2+\varepsilon)}, \delta\right\} .
$$

Assume for simplicity that $\bar{x}=0$ is a local minimizer for $\varphi_{1}+\varphi_{2}$ with $\varphi_{1}(0)=$ $\varphi_{2}(0)=0$. Let us form two closed sets

$$
\Omega_{1}:=\operatorname{epi} \varphi_{1} \quad \text { and } \quad \Omega_{2}:=\left\{(x, \mu) \in X \times \mathbf{R} \mid \varphi_{2}(x) \leq-\mu\right\} .
$$

One can easily check that $(0,0)$ is a locally extremal point of the system $\left\{\Omega_{1}, \Omega_{2}\right\}$. Therefore, we can apply the $F_{\varepsilon}$-extremal principle to this system at $(0,0)$. 
Using the latter result with parameter $\gamma$ in (4.6), one gets $\left(x_{i}, \mu_{i}\right) \in \Omega_{i}$ and $\left(x^{*}, \lambda\right) \in X^{*} \times \mathbf{R}$ such that $\left\|x_{i}\right\| \leq \gamma,\left|\mu_{i}\right| \leq \gamma$ for $i=1,2$ and

$$
\begin{gathered}
\left(x^{*},-\lambda\right) \in \widehat{N}_{\gamma}\left(\left(x_{1}, \mu_{1}\right) ; \Omega_{1}\right), \quad\left(-x^{*}, \lambda\right) \in \widehat{N}_{\gamma}\left(\left(x_{2}, \mu_{2}\right) ; \Omega_{2}\right), \\
\left\|\tilde{x}^{*}\right\|+|\lambda|=1 / 2 .
\end{gathered}
$$

Now let us prove by contradiction that (4.6)-(4.8) imply $\mu_{1}=\varphi_{1}\left(x_{1}\right), \lambda>0$, and $\mu_{2}=-\varphi_{2}\left(x_{2}\right)$.

Indeed, assuming $\mu_{1}>\varphi_{1}\left(x_{1}\right)$ we get directly from (4.7) and (2.1) that $|\lambda| \leq \gamma$. Furthermore, Lipschitzness of $\varphi_{1}$ implies $\left\|x^{*}\right\| \leq 2 \gamma(L+1)$. This contradicts (4.8) and proves $\mu_{1}=\varphi_{1}\left(x_{1}\right)$ due to the choice of $\gamma<1 /(4 L+6)$ in (4.6). Similarly one can conclude that $\lambda>0$ and, moreover,

$$
1 /(4+L) \leq \lambda \leq 1 / 2
$$

Therefore, we have $\tilde{x}^{*} / \lambda \in \widehat{\partial}_{\gamma / \lambda} \varphi_{1}\left(x_{1}\right)$ from (2.4) and the first inclusion in (4.7). Now Proposition 2.1 implies that $\tilde{x}^{*} / \lambda \in \tilde{\partial}_{\alpha} \varphi_{1}\left(x_{1}\right)$ with

$$
\alpha:=\frac{\gamma / \lambda}{1-(\gamma / \lambda)}\left(1+\frac{\left\|\tilde{x}^{*}\right\|}{\lambda}\right)=\left(\frac{\gamma}{\lambda-\gamma}\right) \frac{1}{2 \lambda} .
$$

Due to (4.9) and (4.6) we conclude that $\alpha<\varepsilon$ and

$$
\tilde{x}^{*} / \lambda \in \widetilde{\partial}_{\varepsilon} \varphi_{1}\left(x_{1}\right) \quad \text { with }\left\|x_{1}\right\| \leq \delta \text { and }\left|\varphi_{1}\left(x_{1}\right)\right| \leq \delta .
$$

Similarly one can ensure that $\mu_{2}=-\varphi_{2}\left(x_{2}\right)$. Now taking into account the structure of the set $\Omega_{2}$, we deduce from the second inclusion in (4.7) and the previous estimations that

$$
-x^{*} / \lambda \in \widetilde{\partial}_{\varepsilon} \varphi_{2}\left(x_{2}\right) \text { with }\left\|x_{2}\right\| \leq \delta \text { and }\left|\varphi_{2}\left(x_{2}\right)\right| \leq \delta .
$$

From (4.10) and (4.11) we get (4.5) and finish the proof of the lemma.

Proof of Theorem 3.2. Let us prove the theorem following the scheme (i) $\Rightarrow$ (ii) $\Rightarrow$ (iii) $\Rightarrow$ (i).

First we assume that (i) is true, i.e., $X$ is an Asplund space. It is well known that the property of Asplundity is invariant with respect to Cartesian products. Therefore, the space $X^{k}$ is Asplund for any natural number $k$. Now using implication (a) $\Rightarrow$ (c) in Proposition 2.2, we conclude that "fuzzy calculus" (2.9) for Fréchet subdifferentials holds in the space $X^{2}$. By virtue of Lemma 4.1 this implies the $F$-extremal principle for any extremal system of two sets in $X$.

Let us consider the general case of $n$ closed sets $\left\{\Omega_{1}, \ldots, \Omega_{n}\right\}$ in $X$ and use induction for $n \geq 2$. It is easy to see that if $\bar{x}$ is a locally extremal point of the system $\left\{\Omega_{1}, \ldots, \Omega_{n}\right\}$, then the point $\bar{z}=(\bar{x}, \ldots, \bar{x}) \in X^{n-1}$ is locally extremal for the system of two closed sets

$$
\Lambda_{1}:=\Omega_{1} \times \ldots \times \Omega_{n-1} \quad \text { and } \quad \Lambda_{2}:=\left\{(x, \ldots, x) \in X^{n-1} \mid x \in \Omega_{n}\right\} .
$$

Therefore, we can employ Lemma 4.1 in the Asplund space $X^{n-1}$ and get the $F$-extremal principle for $\bar{z} \in \Lambda_{1} \cap \Lambda_{2}$. Now taking into account (2.3) and the representation

$$
\widehat{N}\left(\bar{z} ; \Lambda_{2}\right)=\left\{\left(x_{1}^{*}, \ldots, x_{n-1}^{*}\right) \in\left(X^{*}\right)^{n-1} \mid x_{1}^{*}+\ldots+x_{n-1}^{*} \in \widehat{N}\left(\bar{x} ; \Omega_{n}\right)\right\},
$$

we conclude that the $F$-extremal principle holds for $\left\{\Omega_{1}, \ldots, \Omega_{n}\right\}$ at $\bar{x}$. This proves (i) $\Rightarrow$ (ii). The next implication (ii) $\Rightarrow$ (iii) follows directly from (2.2). 
It remains to prove that (iii) $\Rightarrow$ (i). Let us consider elements $e \in X$ with $\|e\|=1, \bar{x}^{*} \in X^{*}$ with $\left\langle\bar{x}^{*}, e\right\rangle \neq 0$ and a "hyperplane" $H \subset X$ defined by $H:=$ $\left\{x \in X \mid\left\langle\bar{x}^{*}, x\right\rangle=0\right\}$. One can easily see that $X=H+\mathbf{R} e$ and the mapping

$$
A x:=(h, \alpha) \text { for } x=h+\alpha e
$$

is a linear isomorphism between $X$ and the space $Y:=H \times \mathbf{R}$ with the norm $\|y\|=\|(h, \alpha)\|:=\|h\|+|\alpha|$. Let us show that the $F_{\varepsilon}$-extremal principle in $X$ implies the one in $Y$.

To establish this, we consider an extremal system of closed sets $\Lambda_{i} \subset Y, i=$ $1, \ldots, n(n \geq 2)$, a locally extremal point $\bar{y}$ of $\left\{\Lambda_{1}, \ldots, \Lambda_{n}\right\}$, and any $\nu>0$. Taking $\bar{x}:=A^{-1} \bar{y}$ and $\Omega_{i}:=A^{-1} \Lambda_{i}$, one can easily see that $\bar{x}$ is a locally extremal point of the system $\left\{\Omega_{1}, \ldots, \Omega_{n}\right\}$ in $X$. Let us apply the $F_{\varepsilon}$-principle to the latter system in $X$ with $\varepsilon=\nu /\|A\|$. In this way, we find points $x_{i} \in \Omega_{i} \cap B_{\nu /\|A\|}(\bar{x})$ and $x_{i}^{*} \in X^{*}$, $i=1, \ldots, n$, satisfying relationships (3.1)-(3.3) with $\varepsilon=\nu /\|A\|$. Now define

$$
y_{i}:=A x_{i}, \quad y_{i}^{*}:=\left(A^{-1}\right)^{*} x_{i}^{*}, \quad \text { and } \quad \gamma:=\left\|y_{1}^{*}\right\|+\ldots+\left\|y_{n}^{*}\right\| .
$$

Obviously, $y_{i} \in B_{\nu}(\bar{y})$ for all $i=1, \ldots, n$. Taking into account (2.1), (3.1), (3.3), $\left\|A^{*}\right\|=\|A\|$ as well as $\|A x\| \geq\|x\|$ for the operator considered, one gets

$$
\gamma \geq 1 /\|A\| \quad \text { and } \quad y_{i}^{*} \in \widehat{N}_{\nu /\|A\|}\left(y_{i} ; \Lambda_{i}\right) \quad \forall i=1, \ldots, n .
$$

Then denoting $\tilde{y}_{i}^{*}:=y_{i}^{*} / \gamma$ and using (4.12), we obtain that

$$
\tilde{y}_{i}^{*} \in \widehat{N}_{\nu}\left(y_{i} ; \Lambda_{i}\right) \quad \forall i=1, \ldots, n
$$

and relationships (3.2) and (3.3) hold for $\left\{\tilde{y}_{1}^{*}, \ldots, \tilde{y}_{n}^{*}\right\}$. So we establish the $F_{\varepsilon^{-}}$ extremal principle in the space $Y=H \times \mathbf{R}$ provided that it holds in $X$.

Now Lemma 4.2 ensures that the $F_{\varepsilon}$-principle in $X$ implies property (4.5) in the space $H$. Let us show that this actually implies "fuzzy calculus" (2.8) in $H$.

Given $\varepsilon>0$ and $\delta>0$, let $x^{*} \in \tilde{\partial}_{\varepsilon}\left(\varphi_{1}+\ldots+\varphi_{n}\right)(\bar{x})$ in the setting (b) of Proposition 2.2 for the space $H$. It follows from (2.6) that $\bar{x}$ provides a local minimum to the function

$$
\varphi_{1}(x)+\ldots+\varphi_{n}(x)-\left\langle x^{*}, x\right\rangle+\eta \varepsilon\|x-\bar{x}\|, \quad x \in H,
$$

for any fixed $\eta>1$. Let us apply Lemma 4.2 with $\varepsilon:=\beta \varepsilon, \beta \leq 1 / 2 \eta$, to the sum of functions in (4.13). In this way, we find points $x_{i} \in B_{\delta}(\bar{x})$ with $\left|\varphi_{i}\left(x_{i}\right)-\varphi_{i}(\bar{x})\right| \leq \delta$ such that

$$
x^{*} \in \widetilde{\partial}_{\varepsilon} \varphi_{1}\left(x_{1}\right)+\ldots+\widetilde{\partial}_{\varepsilon} \varphi_{n}\left(x_{n}\right)+(\beta+\eta) \varepsilon B^{*} \subset \widetilde{\partial}_{\varepsilon} \varphi_{1}\left(x_{1}\right)+\ldots+\widetilde{\partial}_{\varepsilon} \varphi_{n}\left(x_{n}\right)
$$

by virtue of (2.6), $\eta>1$, and the choice of $\beta$. Thus we get "fuzzy calculus" (2.8) in the space $H$ that implies Asplundity of $H$ by virtue of Proposition 2.2. Therefore, we can conclude that both spaces $H \times \mathbf{R}$ and $X$ are Asplund since the property of being an Asplund space is preserved under Cartesian products and linear isomorphisms. This establishes $($ iii $) \Rightarrow(\mathrm{i})$ and ends the proof of the theorem.

\section{ACKNOWLEDGMENTS}

We are indebted to Jon Borwein and Marian Fabian for fruitful discussions on this material and to anonymous referee for valuable remarks. 


\section{REFERENCES}

1. E. Asplund, Fréchet differentiability of convex functions, Acta Math. 121 (1968), 31-47. MR 37:6754

2. J. M. Borwein and D. Preiss, A smooth variational principle with applications to subdifferentiability and to differentiability of convex functions, Trans. Amer. Math. Soc. 303 (1987), 517-527. MR 88k:49013

3. J. M. Borwein and H. M. Strojwas, Proximal analysis and boundaries of closed sets in Banach spaces, part II: Applications, Canad. J. Math. 39 (1987), 428-472. MR 88f:46034

4. R. Deville, G. Godefroy, and V. Zizler, Smoothness and renorming in Banach spaces, Pitman Monographs Surveys Pure Appl. Math., vol. 64, Wiley, New York, 1993. MR 94d:46012

5. I. Ekeland, On the variational principle, J. Math. Anal. Appl. 47 (1974), 324-358. MR 49:11344

6. M. Fabian, Subdifferentials, local $\varepsilon$-supports and Asplund spaces, J. London Math. Soc. 34 (1986), 568-576. MR 88a:90216

7. _ Subdifferentiability and trustworthiness in the light of a new variational principle of Borweiss and Preiss, Acta Univ. Carolinae 30 (1989), 51-56. MR 91c:49024

8. A. D. Ioffe, On subdifferentiability spaces, Ann. New York Acad. Sci. 410 (1983), 107-119. MR 86g:90124

9. __ Proximal analysis and approximate subdifferentials, J. London Math. Soc. 41 (1990), $175-192$.

10. A. Y. Kruger, Generalized differentials of nonsmooth functions and necessary conditions for an extremum, Siberian Math. J. 26 (1985), 370-379. MR 86j:49038

11. A. Y. Kruger and B. S. Mordukhovich, Extremal points and Euler equations in nonsmooth optimization, Dokl. Akad. Nauk BSSR 24 (1980), 684-687. (Russian) MR 82b:90127

12. B. S. Mordukhovich, Maximum principle in the problem of time optimal control with nonsmooth constraints, J. Appl. Math. Mech. 40 (1976), 960-969. MR 58:7284

13. __ Metric approximations and necessary optimality conditins for general classes of nonsmooth extremal problems, Soviet Math. Dokl. 22 (1980), 526-530.

14. - Approximation methods in problems of optimization and control, Nauka, Moscow, 1988. (Russian)

15. Generalized differential calculus for nonsmooth and set-valued mappings, J. Math. Anal. Appl. 184 (1994), 250-288. CMP 94:11

16. B. S. Mordukhovich and Y. Shao, Nonsmooth sequential analysis in Asplund spaces, Trans. Amer. Math. Soc. (to appear).

17. R. R. Phelps, Convex functions, monotone operators and differentiability, 2nd ed., Lecture Notes in Math., vol. 1364, Springer-Verlag, Berlin, 1993. MR 94f:46055

Department of Mathematics, Wayne State University, Detroit, Michigan 48202

E-mail address: boris@math. wayne.edu 